\title{
Multiple Fano resonances in bimetallic layered nanostructures
}

\author{
Adnan Daud Khan
}

Received: 12 October 2013/Accepted: 29 May 2014/Published online: 25 June 2014

(C) The Author(s) 2014. This article is published with open access at Springerlink.com

\begin{abstract}
Plasmonic Fano resonances arise in bimetallic layered nanostructures (metal-dielectric-metal and dielectric-metal-dielectric-metal) are studied theoretically as the function of their geometrical parameters. Multiple Fano resonances are generated in these nanostructures where several subradiant dark modes appear due to the geometrical symmetry breaking induced by offsetting different layers. The plasmonic responses of the proposed nanoparticles with equal volumes are compared and multiple Fano resonances with large modulation depths are obtained in metal-dielectric-metal structure which is highly suitable for multi-wavelength sensing and plasmon line shaping. However, the dielectric-metal-dielectricmetal nanostructure is found to provide a slightly better tunability of higher-order plasmonic modes compared to metal-dielectric-metal nanostructure due to which it can be useful for biomedical applications.
\end{abstract}

Keywords Fano-like resonance - Plasmons - Multilayer nanoparticles $\cdot$ Symmetry breaking $\cdot$ Surface charge distribution

$\begin{array}{ll}\text { Abbreviations } \\ \text { EIT } & \text { Electromagnetically induced transparency } \\ \text { SERS } & \text { Surface-enhanced Raman spectroscopy } \\ \text { MNS } & \text { Multilayered nanoshell } \\ \text { DNS } & \text { Double nanoshells } \\ \text { FEM } & \text { Finite Element Method } \\ \text { PML } & \text { Perfectly matched layer } \\ \text { DDF } & \text { Dipole-dipole Fano resonance }\end{array}$

A. D. Khan (ه)

Department of Electrical Engineering, Sarhad University of

Science and Information Technology, Peshawar, Pakistan

e-mail: adnandaudkhan@gmail.com

$\begin{array}{ll}\text { CMNS } & \text { Concentric multilayered nanoshell } \\ \text { NC-MNS } & \text { Non-concentric core multilayered nanoshell } \\ \text { MNS-NG } & \text { Multilayered nanoegg } \\ \text { DQF } & \text { Dipole-quadrupole Fano resonance } \\ \text { DOF } & \text { Dipole-octupole Fano resonance } \\ \text { NC-DNS } & \begin{array}{l}\text { Non-concentric double nanoshells } \\ \text { NC-INS } \\ \text { Double nanoshells with non-concentric inner } \\ \text { nanoshell } \\ \text { NC-SRINS }\end{array} \\ \begin{array}{l}\text { Double nanoshells with non-concentric } \\ \text { symmetry reduced inner nanoshell }\end{array} \\ \text { DNS-NG } & \text { Double nanoshells nanoegg }\end{array}$

\section{Introduction}

Fano resonances have been studied extensively in quantum systems, and were realized well in plasmonic nanostructures in recent years. These resonances usually arise from the coupling and interference of a non-radiative mode and a continuum of radiative electromagnetic waves and are distinguished from their Lorentzian counterpart by a distinctive asymmetric line shape. They are typically more sensitive to the size and shape of the nanoparticle and changes of the refractive index of the environment. The plasmonic Fano-like resonances exhibit sharp resonance and strong light confinement and are analogues of electromagnetically induced transparency (EIT) [1]. They have been observed in various symmetric and asymmetric metallic nanostructures that include nanoshells [2-6], multilayered nanocones [7], rings [8-12], dimers [13-20], trimers [21, 22], quadrumer [23, 24], pentamers [25, 26], hexamer [27], and nanoparticle chains [28, 29]. Among all the nanostructures, the plasmonic layered 
nanostructures have attracted many researchers attention because these structures exhibiting multiple Fano resonances over the entire visible spectrum as well as near to mid infrared region. Multiple Fano resonances observed in such nanostructures possess several dark subradiant modes and can simultaneously modify the plasmon line at several spectral positions that are very suitable for multiwavelength surface-enhanced Raman spectroscopy (SERS) compared to a single Fano resonance [7, 30, 31]. The plasmon oscillations of layered nanoparticles can be explained in terms of the interaction between the plasmon modes of the single parts of the nanostructure, as they act one by one. For instance, the plasmon modes of a single metallic nanoshell, composed of a dielectric core and a metallic layer, can be considered as arising from the interaction between the dipolar mode of the metallic sphere $|S\rangle$ and the dipolar mode of the dielectric cavity $|C\rangle$. The hybridization of the sphere and cavity plasmons causes the plasmon to split into higher energy antibonding mode $|+\rangle$ and the lower-energy bonding mode $|-\rangle$. The $|+\rangle$ mode is anti-symmetric dark subradiant mode because it originates from the anti-symmetric coupling between $|S\rangle$ and $|C\rangle$ modes while $|-\rangle$ mode is symmetric bright superradiant mode because it arises from the symmetric interactions between the two modes. The similar conception of hybridization can be applied for a structure which contains more than two layers of metals and dielectrics $[6,20]$. The interactions between different layers can be highly increased by breaking the symmetry of the nanostructure as reported in [4, 32, 33]. Due to symmetry relaxation, distinct higher-order subradiant dark modes appear in the spectrum which will couple to the bright superradiant mode and engenders multiple Fano resonances due to destructive interference.

The focus of this work is to examine theoretically multiple Fano resonances in two kinds of layered plasmonic nanostructures; the gold-silica-gold multilayered nanoshell (MNS) and double nanoshells (DNS). The MNS contains a spherical metallic core, a dielectric spherical shell, and an external metal spherical shell while the DNS contain two alternative layers of metal and dielectric. Higher-order tunable Fano resonances with large modulation depth are obtained in both the MNS and DNS nanostructures for the symmetric and asymmetric cases. The multiple Fano resonances in DNS nanostructure are obtained for the first time in this paper. Previous study on DNS nanostructures was restricted only to single Fano resonance that arises in the symmetric case. Here, the influence of symmetry breaking over the optical response of DNS nanostructure is studied for the first time to achieve multiple Fano resonances. The optical response of both the MNS and DNS nanoparticles is compared at the end and the MNS is found to exhibit Fano resonances with large modulation depth. These asymmetric Fano line shapes with steep variations are typically suitable for three-dimensional plasmon ruler [34], Plasmon-Induced Transparency (PIT) [35], switching [36], multi-wavelength SERS, and plasmon line shaping by modifying the plasmon line at various spectral locations simultaneously [7, 30].

I studied the scattering spectra, the distribution of the induced surface (free and bond) charges and the near-field enhancement of the proposed nanostructures. All the calculations are carried out by COMSOL Multiphysics software with the RF module. COMSOL Multiphysics is commercially available software based on Finite Element Method (FEM), which correctly solves electromagnetic problems at the nanoscale level. Both the near- and farfield optical properties were calculated in the frequency domain using the scattered-field formulation. The 3D simulation space is composed of a nanoparticle, an embedding medium and a perfectly matched layer (PML). The PML eliminates the reflections at the domain boundaries. The experimental material data (Johnson and Christy model) are utilized for the dielectric constant of the gold [37]. The permeability of the metal is $\mu=1$. A spherical far-field integration boundary is brought in between the inner PML boundary and the nanostructure. We chose the dimensions of the embedding volume and the PML in such a way that varying the size of the nanoparticle would not influence the simulation results. A free built-in meshing algorithm in COMSOL is used to discretize the simulation space, which will divide the simulation space into a set of tetrahedral finite elements. A plane wave, used for excitation, is inserted on the inside of PMLs surrounding the embedding medium. The embedding medium is considered air for all cases.

\section{Results and discussion}

\section{Multilayered nanoshell (MNS)}

Multilayered nanoshell (MNS) contains a spherical metallic core, a dielectric spherical shell and an external metal spherical shell. This type of nanostructure starts to become the focus of strong attention in chemical and bimolecular sensing, lasing and SERS [4, 38]. The MNS nanostructure has also the ability to generate higher-order tunable Fano resonances, which could find applications in plasmon line shaping and multi-wavelength SERS [4, 5]. The geometries of a concentric and non-concentric gold-silica-gold MNS are illustrated in Fig. 1a-c. The dimensions of MNS are $R_{1}=30 / R_{2}=45 / R_{3}=70 \mathrm{~nm}$, respectively. The illuminating electromagnetic wave is linearly polarized; the electric field is directed along $x$-axis and the wave propagates in the $z$-direction. 


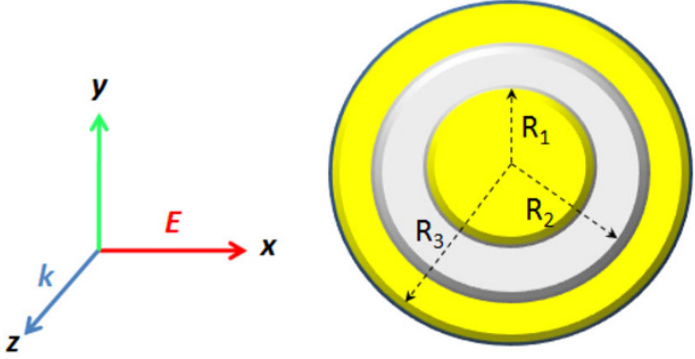

(a)

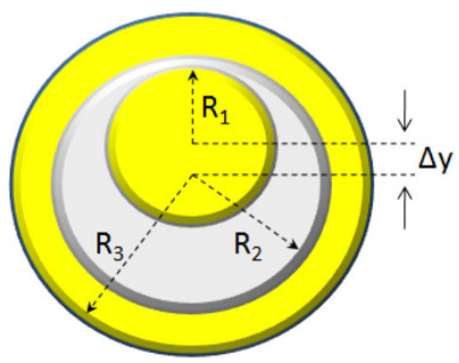

(b)

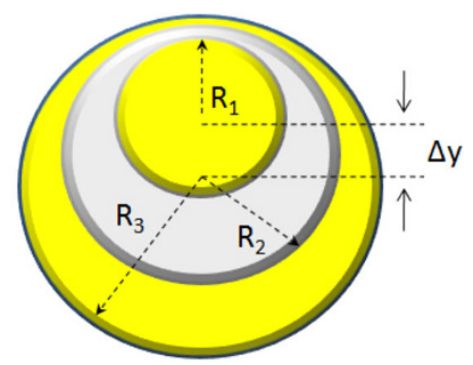

(c)

Fig. 1 Sketch of a concentric MNS. b Non-concentric MNS with offset core in the $y$-direction ( $\Delta y)$ and $\mathbf{c}$ MNS nanoegg with two components offset in the $y$-direction $(\Delta y) . R_{1} / R_{2} / R_{3}$ represents the radius of the inner gold core, middle silica layer and outer gold shell

Fig. 2 Scattering cross-section of a concentric gold-silica-gold MNS

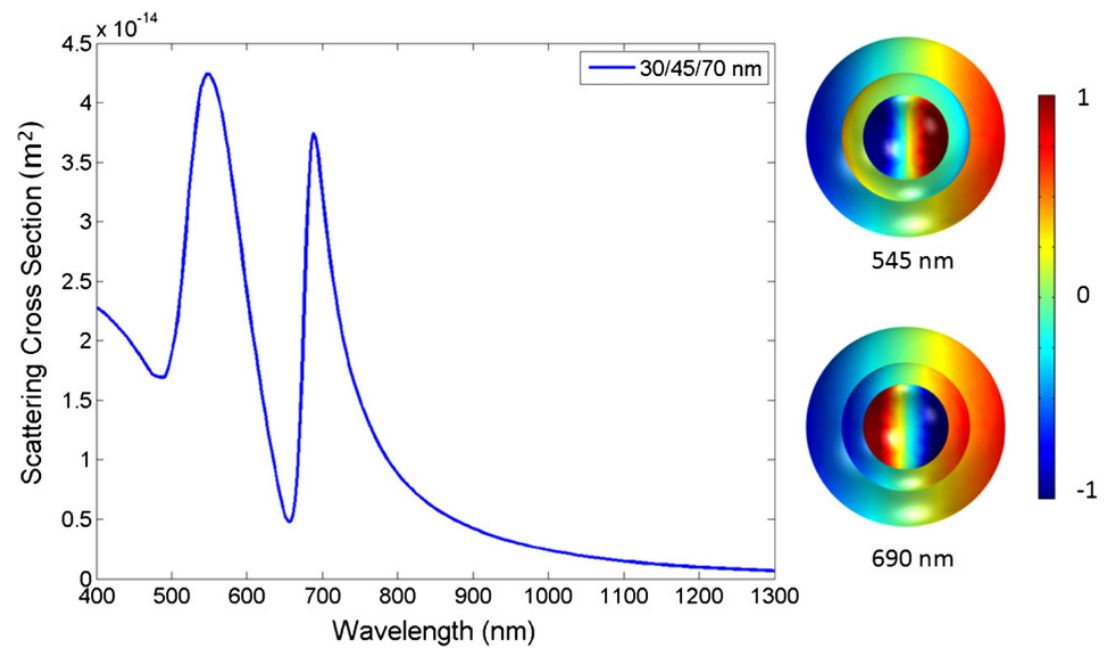

\section{Optical properties of MNS}

The plasmon hybridization model is developed to understand the plasmonic response of complex metallic nanostructures of arbitrary shape [39]. For MNS, the plasmon hybridization will occur between a spherical metallic core and the outer nanoshell. Only the low-energy bonding mode $\left|\omega_{-}\right\rangle$of the nanoshell is considered to interact with the dipole mode of the core because it has a large dipole moment. The high-energy antibonding mode $\left|\omega_{+}\right\rangle$of the nanoshell has a small dipole moment due to which it cannot be observed in the spectrum. Thus, the interaction between the dipole mode of the core and the dipole bonding mode of the shell hybridized and induce a high-energy symmetric dipole antibonding mode $\left|\omega_{-}^{+}\right\rangle_{(1)}$ and a low-energy antisymmetric dipole bonding mode $\left|\omega_{-}^{-}\right\rangle_{(1)}$ as shown by the scattering spectra of a concentric MNS (CMNS) (Fig. 2). The $\left|\omega_{-}^{+}\right\rangle_{(1)}$ mode near $550 \mathrm{~nm}$ is a superradiant mode that arises by the symmetric coupling between the core dipole mode $\left|\omega_{s}\right\rangle$ and the dipole bonding mode $\left|\omega_{-}\right\rangle$of the outer nanoshell, while $\left|\omega_{-}^{-}\right\rangle_{(1)}$ mode (690 nm) which emerges in the vicinity of broad resonance is a subradiant mode that arises by the anti-symmetric coupling between $\left|\omega_{s}\right\rangle$ and $\left|\omega_{-}\right\rangle$modes. The near-field coupling between the two hybridized modes induces a dipole-dipole Fano resonance (DDF) in the spectrum near $655 \mathrm{~nm}$. The surface charge distributions corresponding to the two modes are also shown in the inset where for $\left|\omega_{-}^{+}\right\rangle_{(1)}$ mode, the individual dipole moments of the core and shell oscillate in phase and for $\left|\omega_{-}^{-}\right\rangle_{(1)}$ mode, the dipole modes oscillate out of phase. Due to orthogonality in concentric nanostructures, the plasmon hybridization between different multipolar resonances in the shell and the core is forbidden.

\section{Geometrical tunability of CMNS}

Next, the effect of nanoparticle size on the optical properties has been analyzed, as this parameter plays a crucial role in the occurrence or non-occurrence of DDF (Fig. 3). In Fig. 3a, when $R_{1}$ is small, the gap between the inner core and outer shell becomes large due to which the plasmon coupling between them is weak resulting in a blue-shift of 


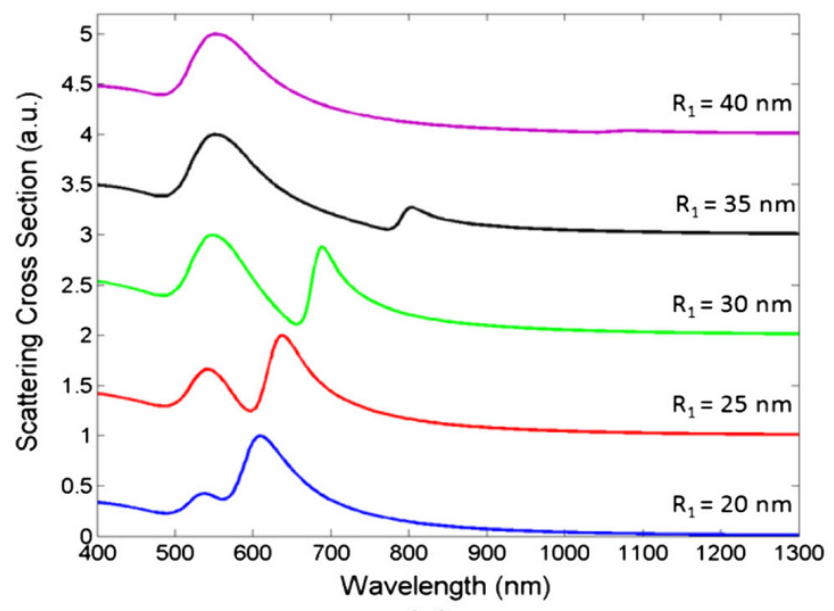

(a)

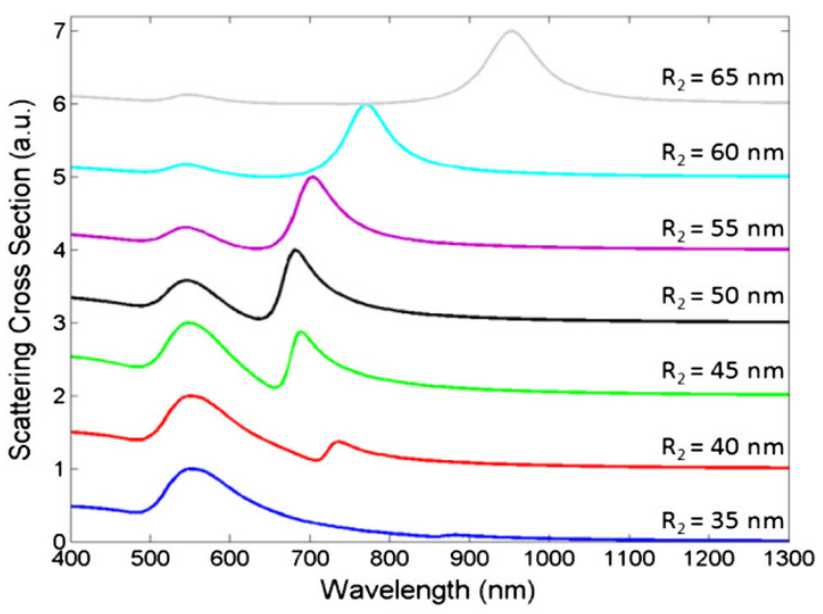

(b)

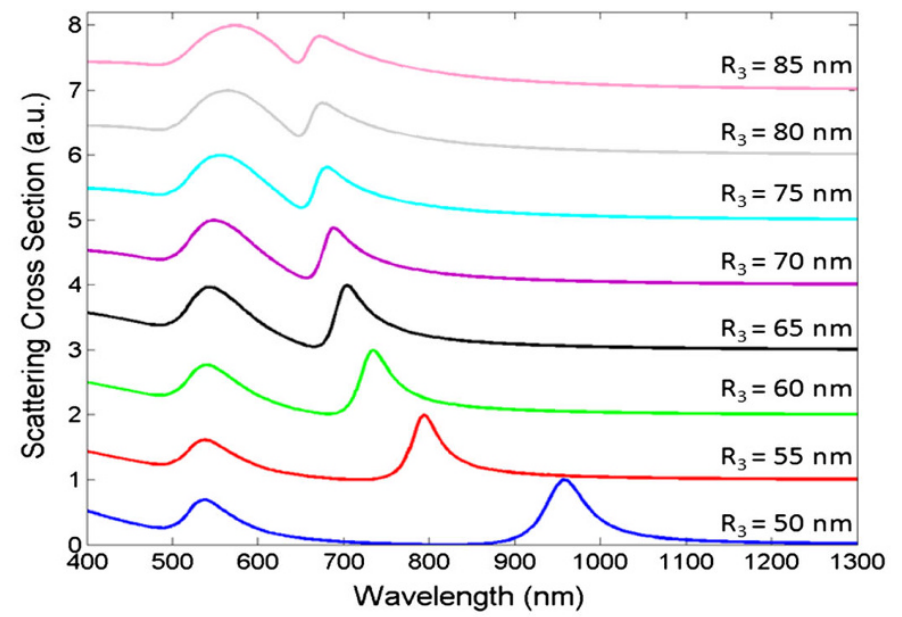

(c)

Fig. 3 Scattering spectra of CMNS for different values of a $R_{1}, \mathbf{b} R_{2}$ and $\mathbf{c} R_{3}$

the DDF and vice versa. For very small gap, both the $\left|\omega_{-}^{+}\right\rangle_{(1)}$ and $\left|\omega_{-}^{-}\right\rangle_{(1)}$ modes are observed far away from each other, they couple directly to the incident light and no Fano resonance is obtained. In Fig. 3b, large value of $R_{2}$ leads to decrease in the plasmon interactions between the gold core and shell due to which $\left|\omega_{-}^{-}\right\rangle_{(1)}$ mode appears blue-shifted. This will enhance the coupling between symmetric and anti-symmetric modes and a sharp DDF arises in the spectrum for $R_{2}=45 \mathrm{~nm}$. By further increasing the value of $R_{2}$, the $\left|\omega_{-}^{-}\right\rangle_{(1)}$ mode shows a redshift due to which the coupling between the modes becomes weak and Fano resonance disappears again. In the last, the influence of the outer gold shell thickness on its optical response is also tested. When $R_{3}$ is small enough than a strong red-shift of the plasmonic peaks in the spectrum are observed because of the stronger interactions between the inner core and the outer shell. Due to smaller
$R_{3}$, both the $\left|\omega_{-}^{+}\right\rangle_{(1)}$ and $\left|\omega_{-}^{-}\right\rangle_{(1)}$ modes are observed to be much far away from each other where they couple directly to the incident field and Fano resonance is absent. For high values of $R_{3}$, a large blue-shift of $\left|\omega_{-}^{-}\right\rangle_{(1)}$ mode is observed. When $R_{3}$ reaches to $60 \mathrm{~nm}$, the coupling between $\left|\omega_{-}^{+}\right\rangle_{(1)}$ and $\left|\omega_{-}^{-}\right\rangle_{(1)}$ modes are established due to which the DDF starts to appear in the spectrum. With further increase of the $R_{3}$ value, the modulation depth of the DDF decreases and also blue-shifted.

Symmetry breaking in MNS

In the previous section, only the symmetric MNS was considered where only those modes which have the same angular momenta interact. For the non-concentric MNS, when the symmetry of the structure is broken, modes of different order will mix i.e., those modes which have 
Fig. 4 Scattering spectra of NC-MNS as a function of core offset $\Delta \mathrm{y} . \Delta y=0$ (blue line), $\Delta y=12 \mathrm{~nm}$ (red line) and $\Delta y=14 \mathrm{~nm}$ (green line $)$
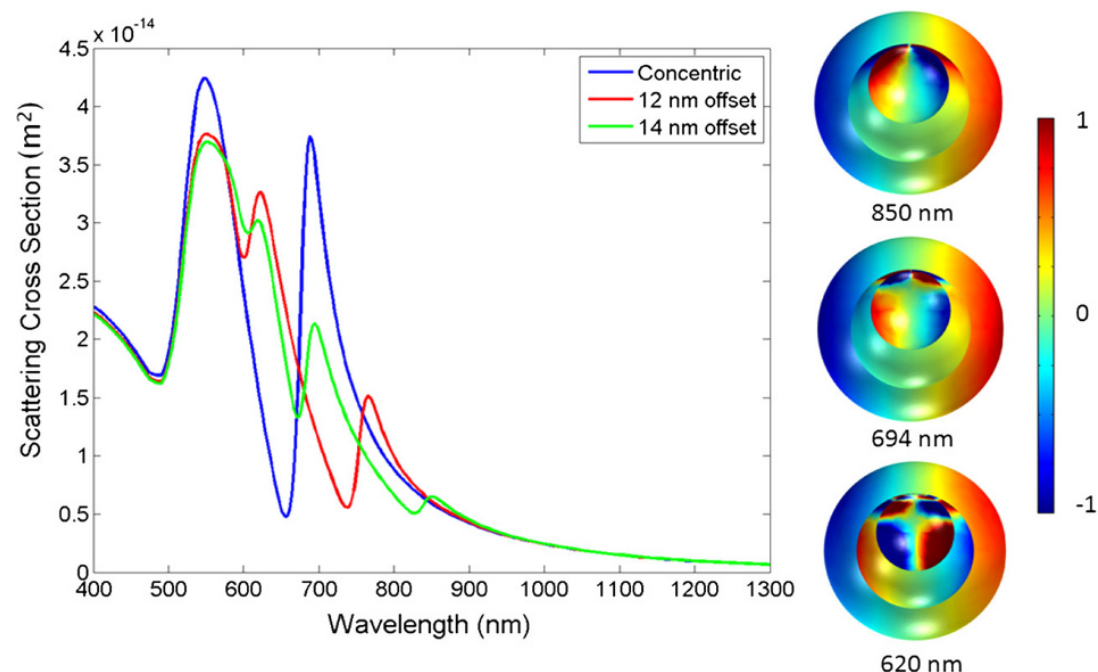

distinctive angular momenta starts to interact [4, 5]. For instance, $\left|\omega_{s}\right\rangle_{(1)}$ mode of the core will not only interact with $\left|\omega_{-}\right\rangle_{(1)}$ mode of the shell but also with the higherorder modes of the shell like the quadrupole $\left|\omega_{-}\right\rangle_{(2)}$ and octupole $\left|\omega_{-}\right\rangle_{(3)}$ modes etc.

Two kinds of symmetry breaking conception are introduced in MNS, first by displacing the inner gold core with respect to the shell (Fig. 1b) and then offsetting both the inner gold core and middle silica layer simultaneously due to which the nanoparticle adopts the shape of a nanoegg (Fig. 1c). The first structure is named as non-concentric core MNS (NC-MNS), while the second structure is named as MNS nanoegg (MNS-NG). Figure 4 shows the scattering spectra of NC-MNS with various core offsets. The blue curve corresponds to the CMNS where I obtained a strong DDF. For a $12 \mathrm{~nm}$ core offset (red curve), a quadrupole mode $\left|\omega_{-}^{-}\right\rangle_{(2)}$ appears in the spectrum, which couple to $\left|\omega_{-}^{+}\right\rangle_{(1)}$ mode and crop up a dipole-quadrupole Fano resonance (DQF) near $600 \mathrm{~nm}$. The DDF red-shifts from $655 \mathrm{~nm}$ to around $740 \mathrm{~nm}$ and the amplitude of $\left|\omega_{-}^{-}\right\rangle_{(1)}$ mode decreases. By further displacing the inner core (14 nm), an octupole mode $\left|\omega_{-}^{-}\right\rangle_{(3)}$ appears in the vicinity of $\left|\omega_{-}^{+}\right\rangle_{(1)}$ mode. The near-field coupling between the two induces a dipole-octupole Fano resonance (DOF) near $605 \mathrm{~nm}$. The DDF and DQF are also red-shifted. Thus, the Fano resonances can be tuned as a function of the core offset. The surface charge distributions corresponding to each hybridized mode are shown in the inset which clearly demonstrates the nature of $\left|\omega_{-}^{-}\right\rangle_{(1)},\left|\omega_{-}^{-}\right\rangle_{(2)}$ and $\left|\omega_{-}^{-}\right\rangle_{(3)}$ modes.

Figure 5 illustrates the scattering spectra of MNS-NG, where I displaced the inner core $37 \mathrm{~nm}$ and middle silica layer $23 \mathrm{~nm}$ from their centers. Five distinct scattering peaks are obtained in the spectrum. By observing the surface charge distributions, the surface charges near $1,071 \mathrm{~nm}$ show a subradiant dipole mode because both the inner core and outer shell exhibit a dipolar pattern. The surface charges near $790 \mathrm{~nm}$ present a quadrupole-quadrupole mode because both the inner core and outer shell reveal a quadrupolar pattern. Since in this nanostructure, the symmetry of the nanoshell is also broken so higherorder modes will also appear on the outer shell due to strong hybridization. The surface charges near $667 \mathrm{~nm}$ show a mixture of octupole modes, while the surface charge distributions near $600 \mathrm{~nm}$ show a quadrupole pattern on the outer layer and hexadecapolar distribution on the inner core, so this slight peak is a combination of quadrupole-hexadecapole mode. Thus, the MNS-NG nanostructure provides distinct higher-order modes and multiple Fano resonances.

\section{Double nanoshells (DNS)}

In this section, the double nanoshells (DNS) structure is constructed by inserting a dielectric sphere of $18 \mathrm{~nm}$ radius inside the inner metallic sphere of MNS. So in this way, I obtained bi-dielectric, bimetallic nanostructure having dimensions $\quad R_{1}=18 / R_{2}=30 / R_{3}=45 / R_{4}=70 \mathrm{~nm} \quad$ as shown in Fig. 6a. The polarization and propagation of incident light remain the same as in the previous section.

\section{Optical properties of DNS}

The plasmon hybridization in DNS will occur between the bonding modes $\left|\omega_{-}\right\rangle$of the inner and outer nanoshells forming high-energy symmetric antibonding mode $\left|\omega_{-}^{+}\right\rangle$ 
Fig. 5 Scattering spectra of MNS-NG. Inset shows surface charge distributions corresponding to each peak
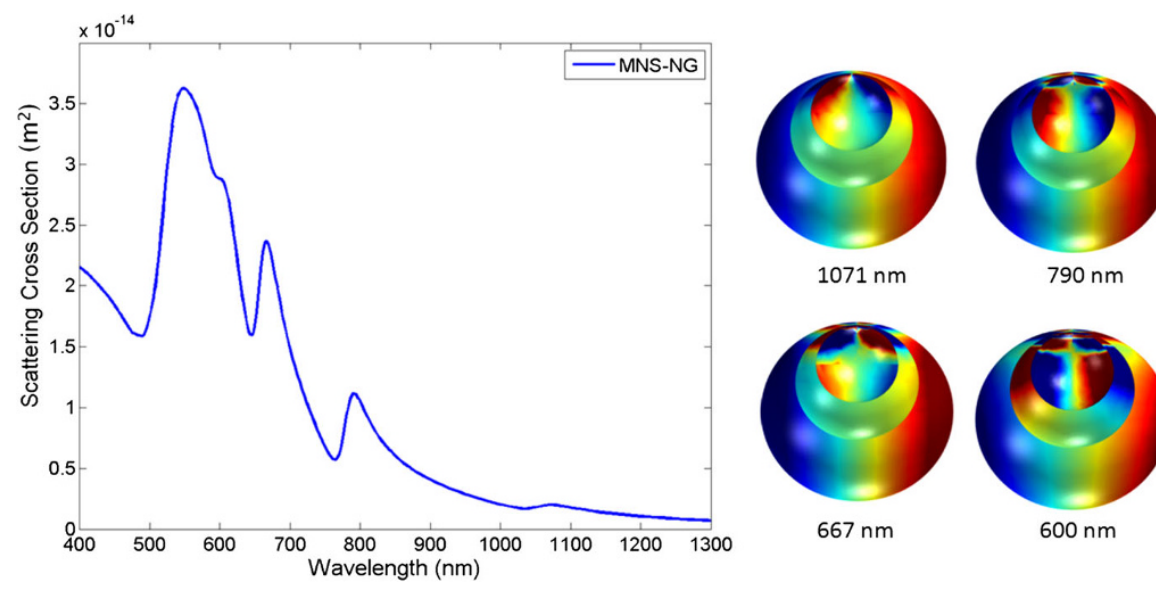

1

$1071 \mathrm{~nm}$

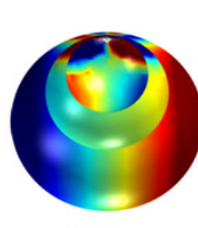

$667 \mathrm{~nm}$

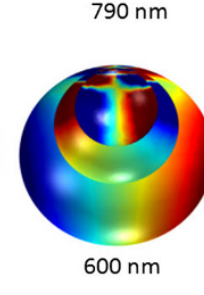

Fig. 6 a Sketch of DNS nanostructure. b Scattering spectra of concentric DNS

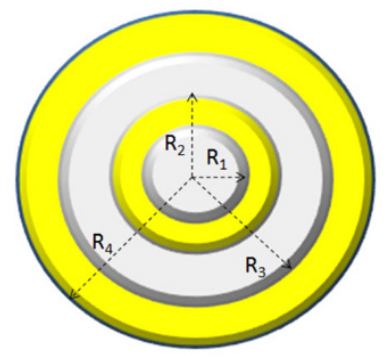

(a)

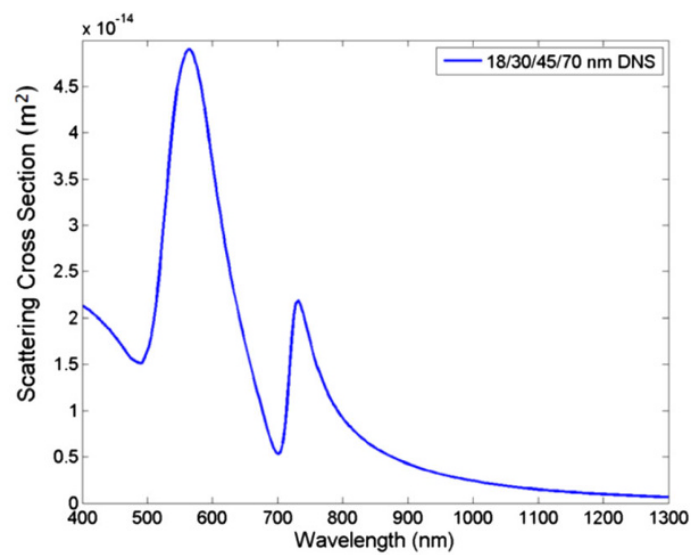

(b) and low-energy anti-symmetric bonding mode $\left|\omega_{-}^{-}\right\rangle$[6]. This is revealed by the scattering spectra of concentric DNS (Fig. 6b) where the high-energy peak near $564 \mathrm{~nm}$ is $\left|\omega_{-}^{+}\right\rangle$mode and the lower-energy peak near $732 \mathrm{~nm}$ is $\left|\omega_{-}^{-}\right\rangle$mode. The dip appears near $700 \mathrm{~nm}$ representing DDF that originates from the near-field coupling between $\left|\omega_{-}^{+}\right\rangle$and $\left|\omega_{-}^{-}\right\rangle$modes. This hybridization seems much similar to the MNS nanostructure.

\section{Geometrical tunability of DNS}

The tunability of DDF in DNS nanostructure is also investigated by varying the nanoparticle size as shown in Fig. 7. Here, additional benefits are obtained compared to MNS nanostructure because of an extra layer due to which this nanostructure has an extra tunable parameter in the aspect ratio of the inner nanoshell. This new parameter typically controls the bonding energy mode of the layered nanostructure. In Fig. 7a, c, the effect of the dielectric spacing layer is studied. By decreasing $R_{1}$, a strong blue- shift of $\left|\omega_{-}^{-}\right\rangle$mode is obtained due to which a Fano resonance with large modulation depth is perceived in the spectrum. By decreasing $R_{3}$, the DDF first blue-shifts and then disappears because $\left|\omega_{-}^{-}\right\rangle$mode strongly red-shifts due to which it does not overlap with $\left|\omega_{-}^{+}\right\rangle$mode. Similarly, in Fig. $7 b, d$, the effects of metallic layer have been studied. The response of modifying $R_{2}$ is nearly similar to $R_{3}$. On the other hand, by increasing $R_{4}$, both the $\left|\omega_{-}^{+}\right\rangle$and $\left|\omega_{-}^{-}\right\rangle$ modes come closer, overlap in energy and induces strong DDF. However, for large values of $R_{4}$, the DDF suffers from weak modulation depth. The DNS nanostructure is found to provide a slightly better tunability of the resonant modes compared to MNS nanostructure because of an extra layer due to which, it can be a better choice for biomedical applications [40].

\section{Symmetry breaking in DNS}

Next, the symmetry of the DNS nanostructure is broken by moving the inner nanoshell in the $y$-direction due to which 


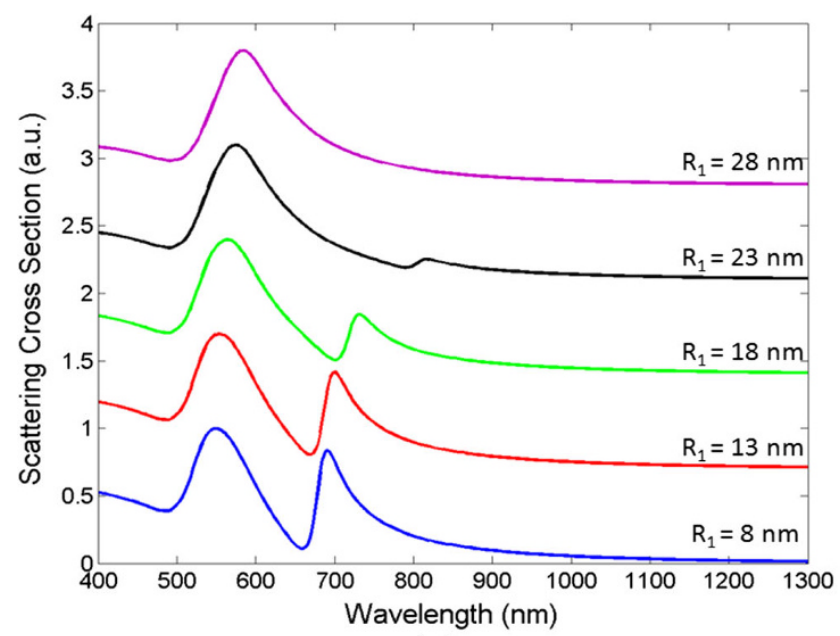

(a)

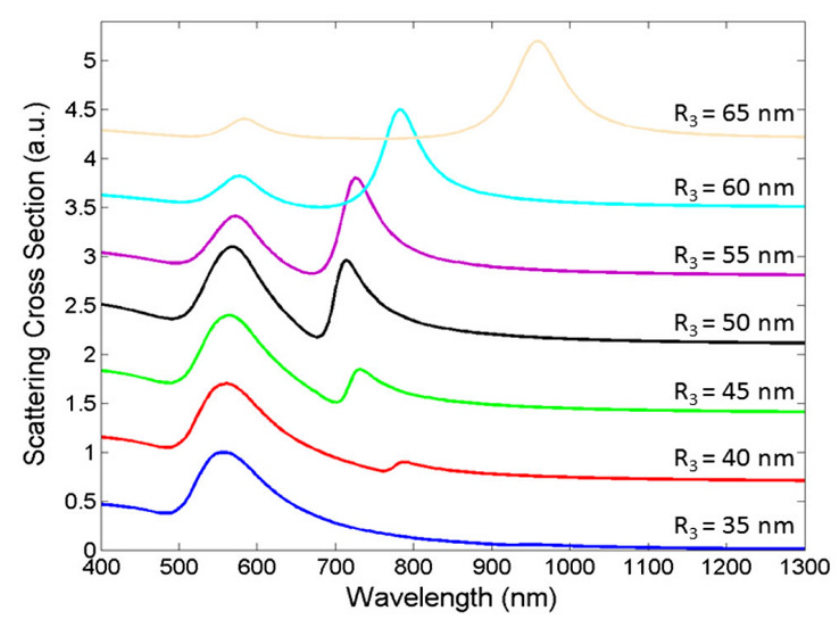

(c)

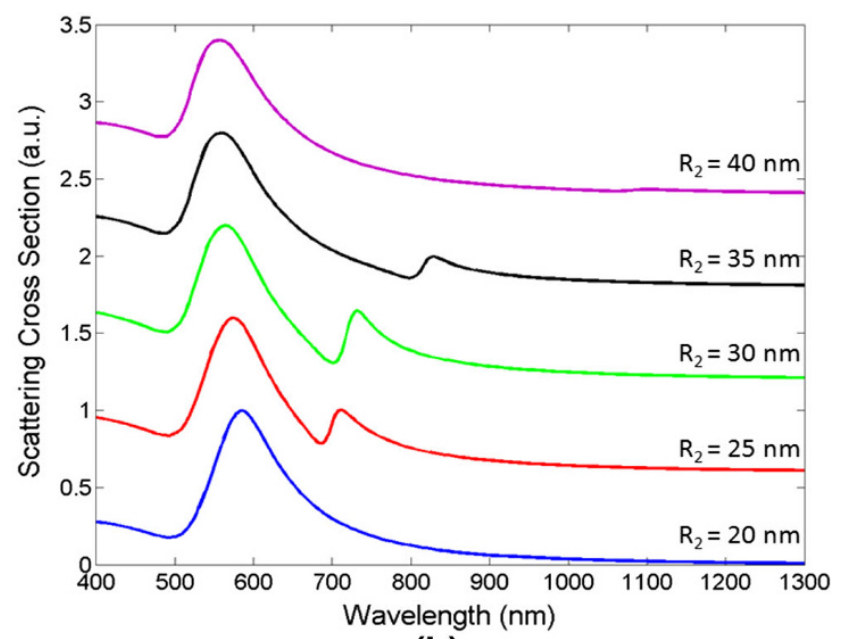

(b)

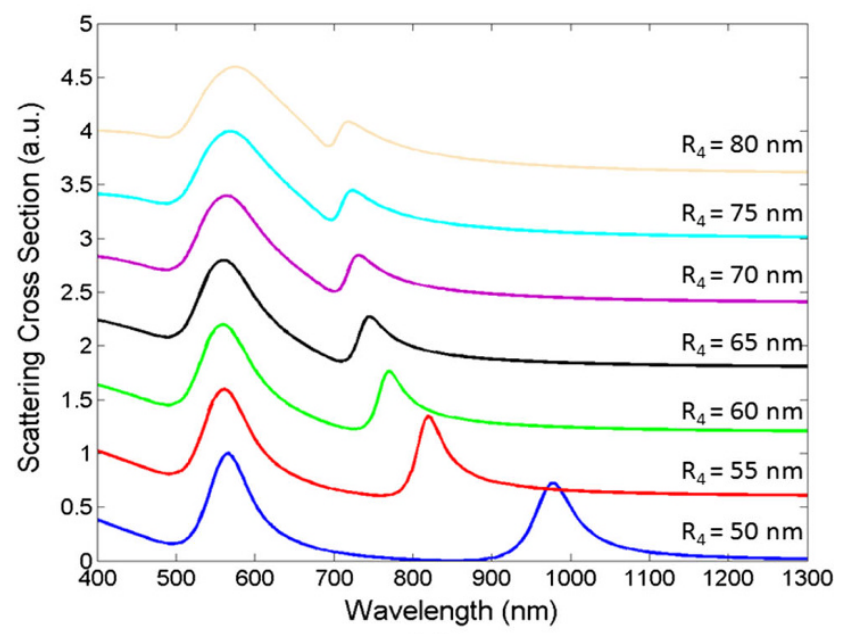

(d)

Fig. 7 Scattering spectra of DNS for different values of $\mathbf{a} R_{1}, \mathbf{b} R_{2}, \mathbf{c} R_{3}$ and $\mathbf{d} R_{4}$

Fig. 8 a Geometry of NC-DNS with inner nanoshell offset $\Delta y$. b Scattering spectra of NCDNS with various inner nanoshell offsets. Blue line $\Delta y=0$, red line $\Delta y=13 \mathrm{~nm}$ and green line $\Delta y=14 \mathrm{~nm}$. Insets shows surface charge distributions for $\Delta y=14 \mathrm{~nm}$

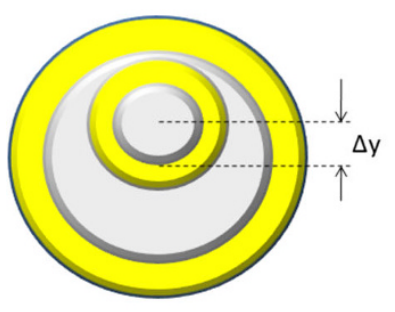

(a)

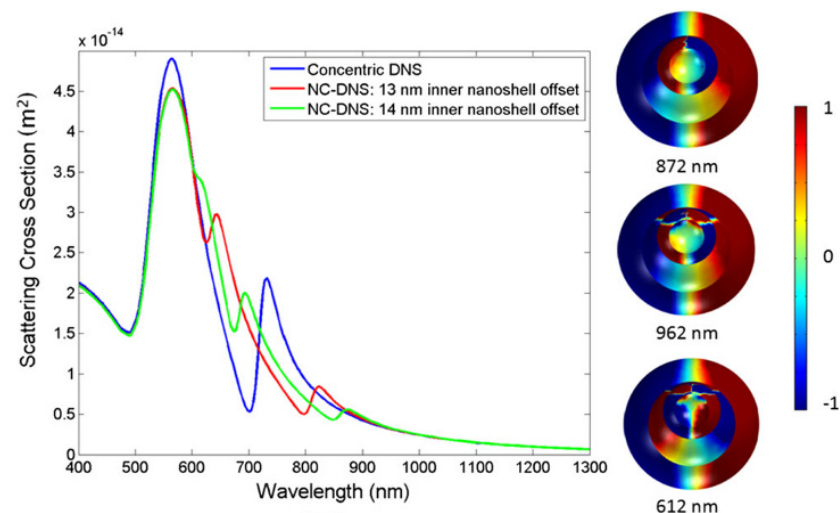

(b) the higher-order hybridized modes come into view like in NC-MNS nanostructure. The geometry of the non-concentric DNS (NC-DNS) is presented in Fig. 8a. Figure 8b shows the scattering spectra of the concentric DNS and NC-DNS with various inner shell offsets. For the concentric DNS, $\left|\omega_{-}^{+}\right\rangle_{(1)}$ and $\left|\omega_{-}^{-}\right\rangle_{(1)}$ modes (blue line) are 
obtained. For NC-DNS, higher-order hybridized modes emerge in the spectrum. By observing the surface charges shown in the inset for $14 \mathrm{~nm}$ inner nanoshell offset, it becomes clear that the resonant peak near $872 \mathrm{~nm}$ is a subradiant dipole mode. The surface charge distributions near $692 \mathrm{~nm}$ show a dipole distribution on the outer nanoshell and quadrupole distribution on the inner nanoshell, so this clearly demonstrates a mixed dipole-quadrupole mode. Similarly, the surface charges near $612 \mathrm{~nm}$ (small peak) show the mixing of dipole and octupole modes. So, the NC-DNS exhibit the similar higher-order hybridized modes and Fano resonances like the NC-MNS nanostructure.

Three other types of symmetry breaking in DNS nanostructure are studied. The first one is to displace the inner silica core of the inner nanoshell $11 \mathrm{~nm}$ on an otherwise fixed geometry, the second one is to offset the symmetry broken inner nanoshell $14 \mathrm{~nm}$ on an otherwise fixed geometry and the third one is to offset both the symmetry reduced inner nanoshell $(37 \mathrm{~nm})$ and the silica layer of the outer nanoshell $(23 \mathrm{~nm})$ simultaneously, which adopts the shape of a nanoegg. All the geometries are shown in Fig. 9a-c. I named the first nanostructure as DNS with non-concentric inner nanoshell (NC-INS), the second one as DNS with non-concentric symmetry reduced inner nanoshell (NC-SRINS) and the third one as DNS nanoegg (DNS-NG). The scattering spectra of NCINS, NC-SRINS and DNS-NG nanostructures are presented in Fig. 9d. For NC-INS nanostructure, only subradiant dipole and quadrupole modes are obtained, which are demonstrated by the surface charge distributions in the lower panel. By observing the surface charge distributions of NC-SRINS nanostructure given in the middle panel, the resonant peak near $794 \mathrm{~nm}$ shows the mixed dipolequadrupole character of the dipole-quadrupole mode. The peak near $675 \mathrm{~nm}$ is the mixture of dipole and octupole modes, while the peak near $610 \mathrm{~nm}$ shows a dipole distribution on the outer nanoshell and hexadecapolar distribution on the inner nanoshell depicting dipolehexadecapolar mode. The lower order subradiant dipole mode remains absent in the NC-SRINS nanostructure. For the DNS-NG nanostructure, the higher-order modes will also be appeared on the outer nanoshell because the symmetry of the outer nanoshell is also broken (similar like MNS-NG). Therefore, by examining the surface charge distributions near $872 \mathrm{~nm}$, a quadrupole pattern emerges on both the outer and inner nanoshells, which represent a quadrupole-quadrupole mode. The surface charges near $718 \mathrm{~nm}$ show a combination of octupole modes while the surface charges near $636 \mathrm{~nm}$ exhibit quadrupole distribution on the outer nanoshell and an octupole distribution on the inner nanoshell, which clearly shows the mixed quadrupole-octupole character of the quadrupole-octupole mode. Figure 9e shows the closer view of the surface charges corresponding to DNS-NG and NC-SRINS nanostructures. For all the considered cases, the superradiant bright mode has sustained its spectral position. To our knowledge, the higher-order Fano resonances in DNS nanostructure are never reported before. These multiple Fano resonances could find applications in plasmon line shaping, multi-wavelength SERS, and biosensing.

\section{Comparison of MNS and DNS nanostructures}

The near- and far-field properties of both the MNS and DNS are compared by taking both the nanostructures with equal volumes. Figure 10a shows the scattering spectra of both the nanostructures for a concentric case. A comparatively much stronger Fano resonance with large modulation depth is observed in MNS nanostructure. Figure 10b shows the scattering spectra of both the nanostructures for nonconcentric cases, i.e., the inner metal core in MNS has been offset $47 \%$ from its center and with a similar offset value, the inner nanoshell in DNS has been moved. The figure depicts that both the nanostructures exhibit higher-order Fano resonances; however, the modulation depth of the Fano resonances in case of NC-MNS is observed to be slightly stronger. For instance, the octupole Fano resonance in case of NC-DNS is found to be much weaker compared to NC-MNS nanostructure. Figure 10c shows the scattering spectra of MNS and DNS nanostructures with nanoegg-like shapes, where the symmetry of both the nanostructures is broken with the same degree of offset. The MNS-NG exhibits five scattering peaks in the spectrum compared to DNS-NG nanostructure. Thus, the MNS, NC-MNS and MNS-NG nanostructures have the potential in the generation of higher-order Fano resonances with large modulation depths.

Eventually, the Fano resonances in the near-field optical properties of both the MNS and DNS nanostructures are compared. Figure 11 shows the maximum near-field enhancement spectra of NC-MNS, NC-DNS, MNS-NG and DNS-NG nanostructures. In Fig. 11a, the higher-order hybridized modes and Fano resonances for both the nanostructures are attained almost at the same spectral location as that in the far-field spectra. However, the weak octupole mode is missing in this case. The near-field spectra of both the nanostructures clearly show that NCMNS provides maximum value of the near-field enhancement at various spectral locations compared to NC-DNS nanostructure. Similarly, in Fig. 11b, all the hybridized 


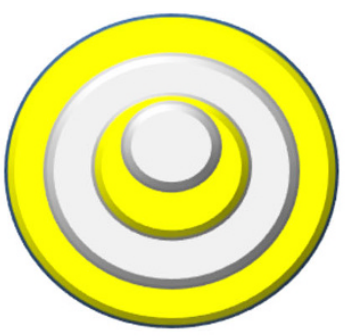

NC-INS

(a)

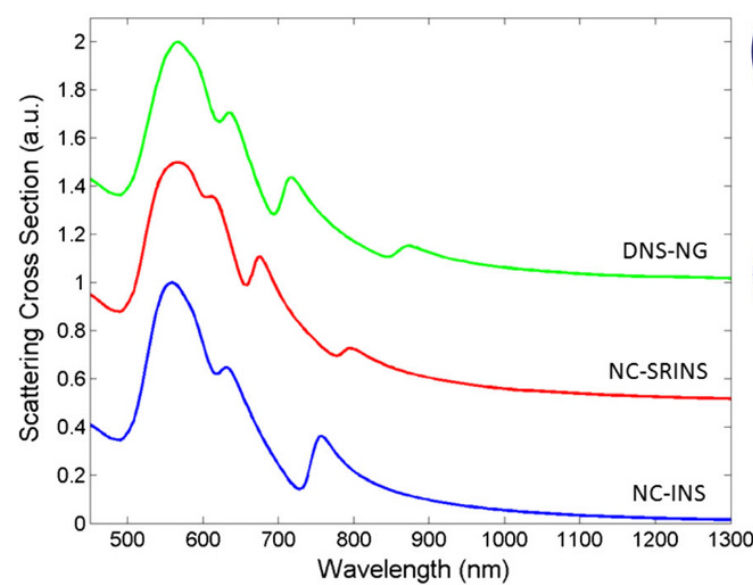

(d)

DNS-NG

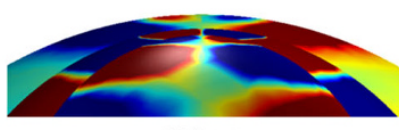

$636 \mathrm{~nm}$

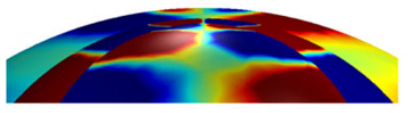

$718 \mathrm{~nm}$

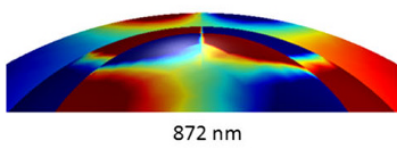

(b)

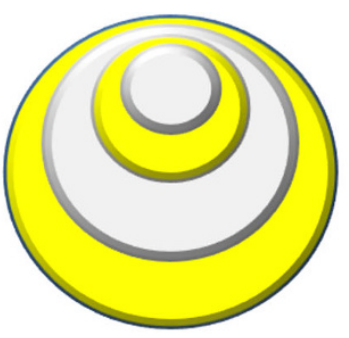

DNS-NG

(c)

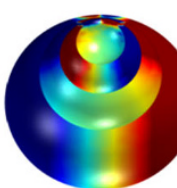

$872 \mathrm{~nm}$

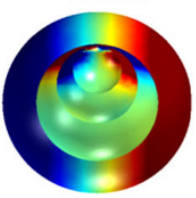

$794 \mathrm{~nm}$

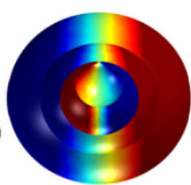

$760 \mathrm{~nm}$

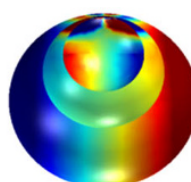

$718 \mathrm{~nm}$

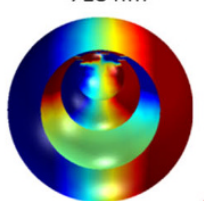

$675 \mathrm{~nm}$

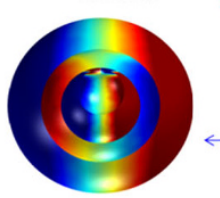

$630 \mathrm{~nm}$

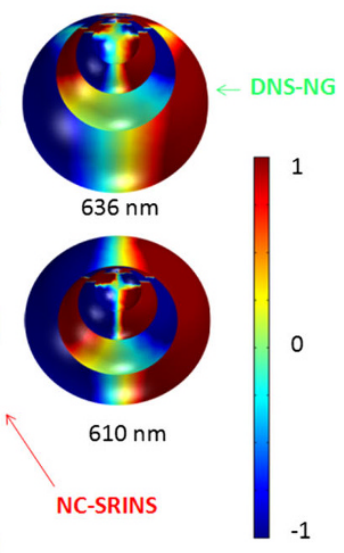

NC-SRINS

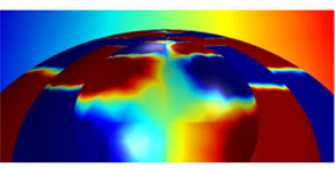

$610 \mathrm{~nm}$

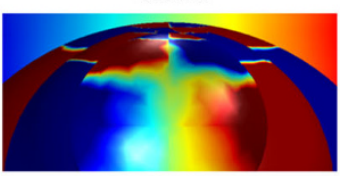

$675 \mathrm{~nm}$

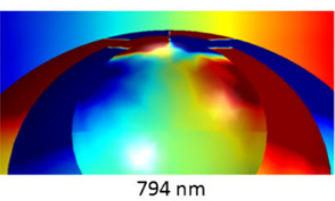

(e)

distributions for NC-INS (bottom panel), NC-SRINS (middle panel) and DNS-NG (top panel) nanostructures. e Closer view of surface charges corresponding to DNS-NG and NC-SRINS nanostructures

enhancement calculations, it has been established that MNS nanostructure shows strong near-field enhancement in several regions compared to DNS nanostructure, which

Fig. 9 Geometric sketches of a NC-INS, b NC-SRINS and c DNSNG nanostructures. d Scattering cross-sections. Blue line corresponds to NC-INS, red line corresponds to NC-SRINS and green line corresponds to DNS-NG nanostructure. Insets show surface charge

modes are achieved successfully in the near-field spectra and both the MNS-NG and DNS-NG nanostructures provide almost similar values of the enhancement. From field 


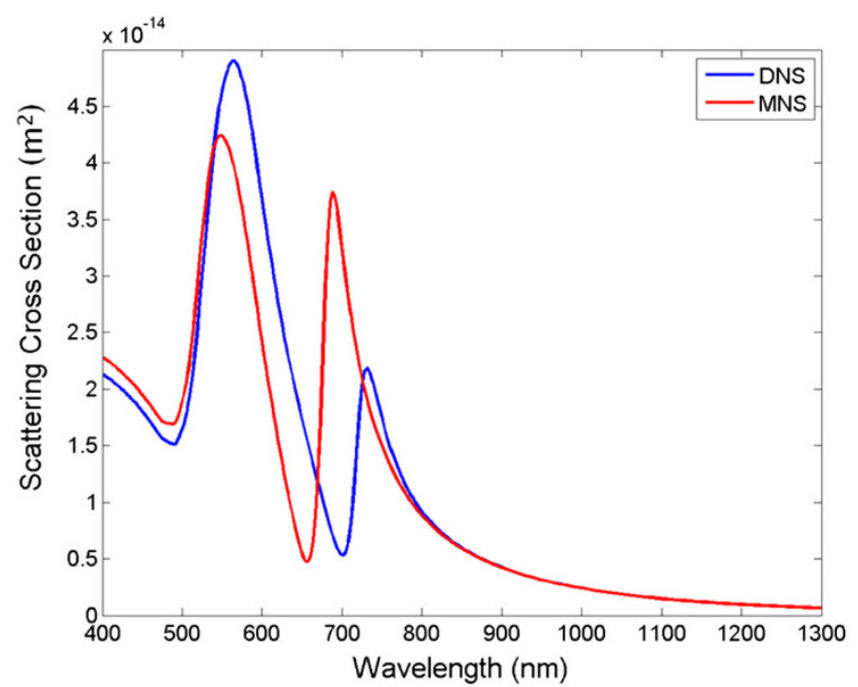

(a)

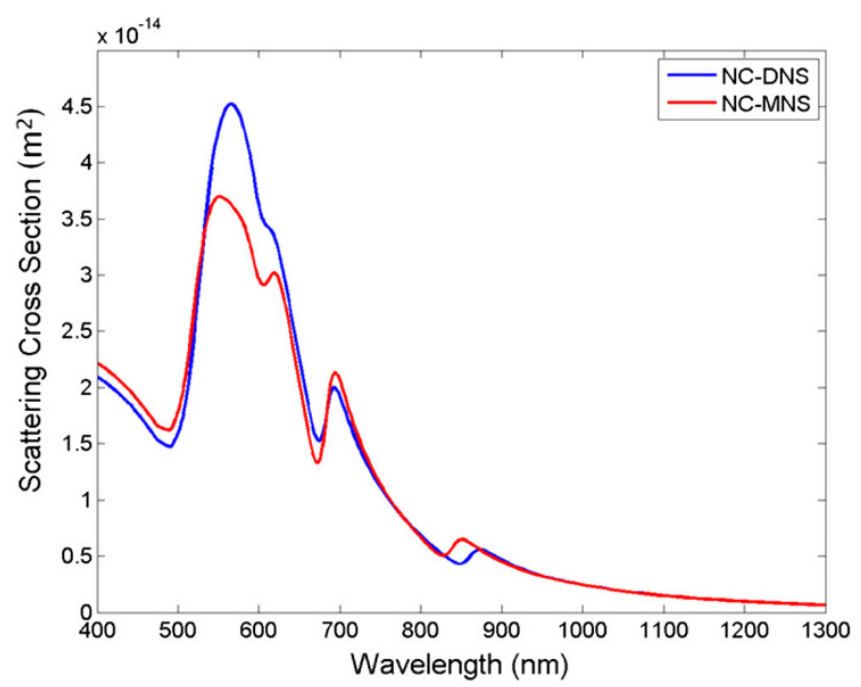

(b)

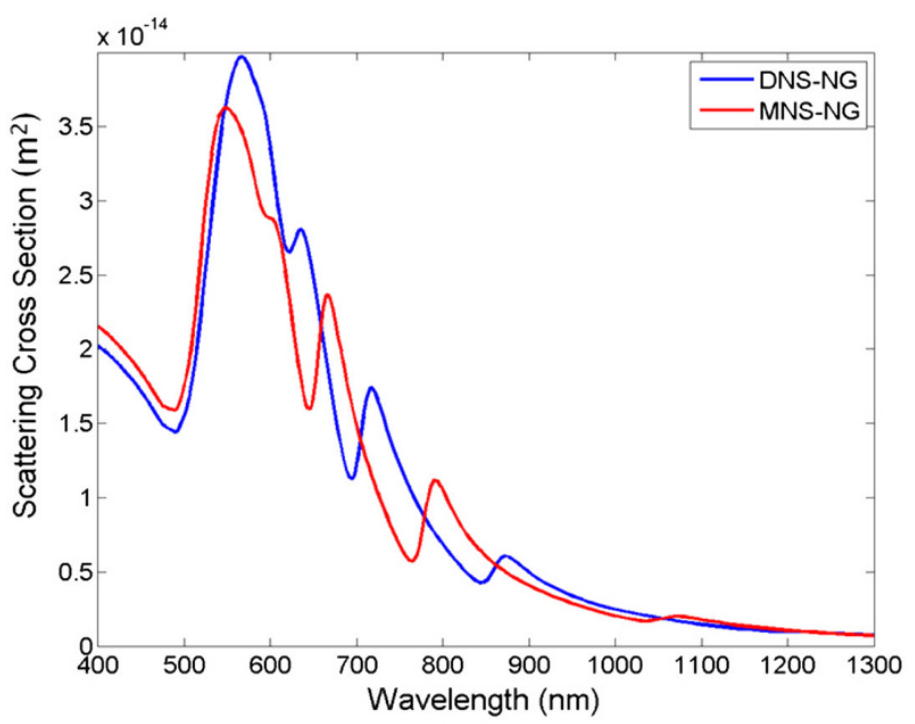

(c)

Fig. 10 Scattering spectra a DNS and MNS nanostructures. b NC-DNS and NC-MNS nanostructures. c DNS-NG and MNS-NG nanostructures

may provide effective applications in surface-enhanced spectroscopy and localized surface plasmon resonance biosensor [41].

\section{Conclusion}

Plasmonic Fano-like resonances in bimetallic layered nanostructures are investigated. It is established that the resonant energy and intensity of Fano resonances can be tuned by changing the geometric parameters of the nanoparticle. Multiple Fano resonances are observed with prominent modulation depth by breaking the symmetry of the structure. The higher-order multiple Fano resonances are also studied for the first time in DNS nanostructure and its comparison is made with MNS nanostructure. The DNS nanostructure is proved to provide a slightly better tunability of plasmonic peaks compared to MNS nanostructure because of the addition of an extra layer due to which it can be useful for biomedical applications. However, the MNS nanostructure is revealed to be a better choice for the generation of higher-order tunable Fano resonances with large modulation depth, particularly suitable for three-dimensional plasmon ruler, multi-wavelength SERS, and plasmon line shaping by simultaneously modifying the plasmon line at several spectral positions. Future work may comprise the 


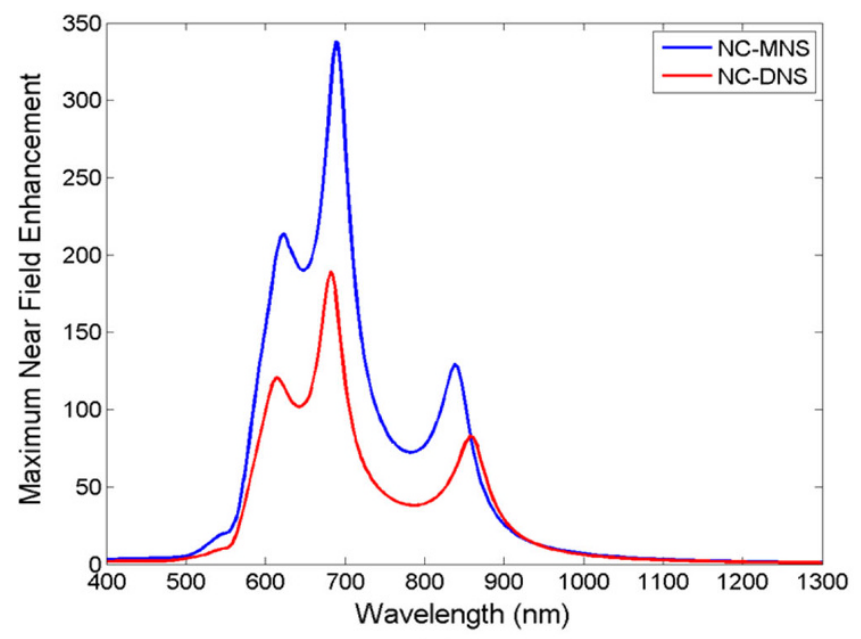

(a)

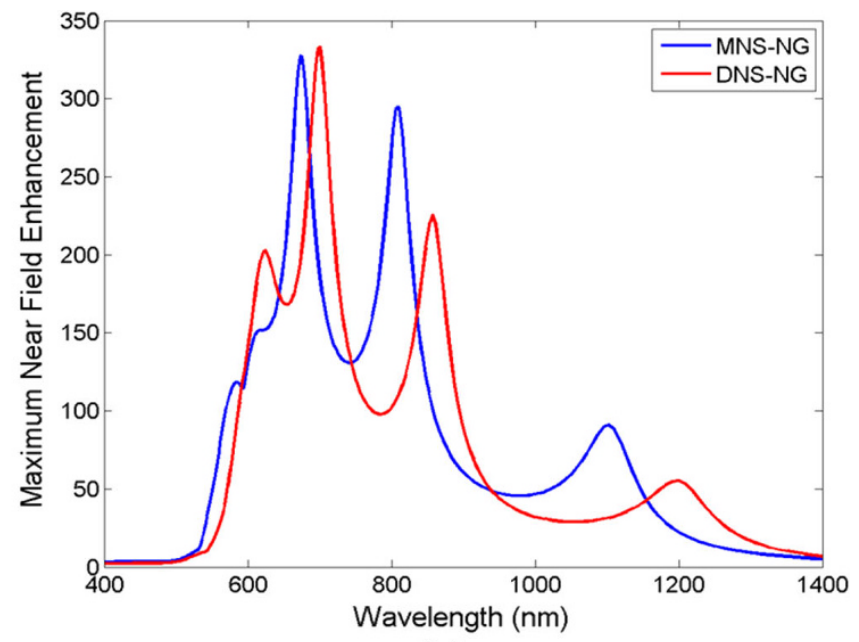

(b)

Fig. 11 Maximum near-field enhancement spectra of a NC-MNC and NC-DNS, b MNS-NG and DNS-NG nanostructures

utilization of other nanoparticle shapes or collective effects in nanoparticle arrays to further increase the generation and tunability of higher-order Fano resonances.

Open Access This article is distributed under the terms of the Creative Commons Attribution License which permits any use, distribution, and reproduction in any medium, provided the original author(s) and the source are credited.

\section{References}

1. Chen, J., Wang, P., Chen, C., Lu, Y., Ming, H., Zhan, Q.: Plasmonic EIT-like switching in bright-dark-bright plasmon resonators. Opt. Express 19(7), 5970-5978 (2011)

2. Khan, A.D., Miano, G.: Plasmonic Fano resonances in single-layer gold conical nanoshells. Plasmonics 8(3), 1429-1437 (2013)

3. Zhang, J., Zayats, A.: Multiple Fano resonances in single-layer nonconcentric core-shell nanostructures. Opt. Express 21(7), 8426-8436 (2013)

4. Mukherjee, S., Sobhani, H., Lassiter, J.B., Bardhan, R., Nordlander, P., Halas, N.J.: Fanoshells: nanoparticles with built-in Fano resonances. Nano Lett. 10(7), 2694-2701 (2010)

5. Ho, J.F., Luk'yanchuk, B., Zhang, J.B.: Tunable Fano resonances in silver-silica-silver multilayer nanoshells. Appl. Phys. A 107(1), 133-137 (2012)

6. Peña-Rodríguez, O., Rivera, A., Campoy-Quiles, M., Pal, U.: Tunable Fano resonance in symmetric multilayered gold nanoshells. Nanoscale 5(1), 209-216 (2013)

7. Khan, A.D., Miano, G.: Higher order tunable Fano resonances in multilayer nanocones. Plasmonics 8(2), 1023-1034 (2013)

8. Shu, J., Gao, W., Xu, Q.: Fano resonance in concentric ring apertures. Opt. Express 21(9), 11101-11106 (2013)

9. He, J., Fan, C., Wang, J., Ding, P., Cai, G., Cheng, Y., Zhu, S., Liang, E.: A giant localized field enhancement and high sensitivity in an asymmetric ring by exhibiting Fano resonance. J. Opt. 15(2), 025007 (2013)

10. Cetin, A.E., Altug, H.: Fano resonant ring/disk plasmonic nanocavities on conducting substrates for advanced biosensing. ACS Nano 6(11), 9989-9995 (2012)
11. Fu, Y.H., Zhang, J.B., Yu, Y.F., Luk'yanchuk, B.: Generating and manipulating higher order Fano resonances in dual-disk ring plasmonic nanostructures. ACS Nano 6(6), 5130-5137 (2012)

12. Zhang, Y., Jia, T., Zhang, H., Xu, Z.: Fano resonances in diskring plasmonic nanostructure: strong interaction between bright dipolar and dark multipolar mode. Opt. Lett. 37(23), 4919-4921 (2012)

13. Khan, A.D., Miano, G.: Investigation of plasmonic resonances in mismatched gold nanocone dimers. Plasmonics (2013). doi:10. 1007/s11468-11013-19595-x

14. Wu, D., Jiang, S., Cheng, Y., Liu, X.: Fano-like resonance in symmetry-broken gold nanotube dimer. Opt. Express 20(24), 26559-26567 (2012)

15. Yang, Z.-J., Zhang, Z.-S., Hao, Z.-H., Wang, Q.-Q.: Fano resonances in active plasmonic resonators consisting of a nanorod dimer and a nano-emitter. Appl. Phys. Lett. 99(8), 081103-081107 (2011)

16. Yang, Z.-J., Zhang, Z.-S., Zhang, L.-H., Li, Q-.Q., Hao, Z.-H., Wang, Q-.Q.: Fano resonances in dipole-quadrupole plasmon coupling nanorod dimers. Opt. Lett. 36(9), 1542-1544 (2011)

17. Xi, Z., Lu, Y., Yu, W., Wang, P., Ming, H.: Improved sensitivity in a T-shaped nanodimer plasmonic sensor. J. Opt. 15(2), 025004 (2013)

18. Peña-Rodríguez, O., Pal, U., Campoy-Quiles, M., RodríguezFernández, L., Garriga, M., Alonso, M.: Enhanced Fano resonance in asymmetrical $\mathrm{Au}$ : Ag heterodimers. J. Phys. Chem. C 115(14), 6410-6414 (2011)

19. Brown, L.V., Sobhani, H., Lassiter, J.B., Nordlander, P., Halas, N.J.: Heterodimers: plasmonic properties of mismatched nanoparticle pairs. ACS Nano 4(2), 819-832 (2010)

20. Khan, A.D., Khan, S.D., Khan, R.U., Ahmad, N.: Excitation of multiple Fano-like resonances induced by higher order plasmon modes in three-layered bimetallic nanoshell dimer. Plasmonics (2013). doi:10.1007/s11468-013-9644-5

21. Wang, J., Fan, C., He, J., Ding, P., Liang, E., Xue, Q.: Double Fano resonances due to interplay of electric and magnetic plasmon modes in planar plasmonic structure with high sensing sensitivity. Opt. Express 21(2), 2236-2244 (2013)

22. Sheikholeslami, S.N., García-Etxarri, A., Dionne, J.A.: Controlling the interplay of electric and magnetic modes via Fano-like plasmon resonances. Nano Lett. 11(9), 3927-3934 (2011) 
23. Fan, J.A., Bao, K., Wu, C., Bao, J., Bardhan, R., Halas, N.J., Manoharan, V.N., Shvets, G., Nordlander, P., Capasso, F.: Fanolike interference in self-assembled plasmonic quadrumer clusters. Nano Lett. 10(11), 4680-4685 (2010)

24. Rahmani, M., Tahmasebi, T., Lin, Y., Lukiyanchuk, B., Liew, T., Hong, M.: Influence of plasmon destructive interferences on optical properties of gold planar quadrumers. Nanotechnology 22(24), 245204 (2011)

25. Rahmani, M., Lukiyanchuk, B., Nguyen, T., Tahmasebi, T., Lin, Y., Liew, T., Hong, M.: Influence of symmetry breaking in pentamers on Fano resonance and near-field energy localization. Opt. Mater. Express 1(8), 1409-1415 (2011)

26. Liu, S.-D., Zhang, M.-J., Wang, W.-J., Wang, Y.-C.: Tuning multiple Fano resonances in plasmonic pentamer clusters. Appl. Phys. Lett. 102(13), 133105 (2013)

27. Mirin, N.A., Bao, K., Nordlander, P.: Fano resonances in plasmonic nanoparticle aggregatest. J. Phys. Chem. A 113(16), 4028-4034 (2009)

28. Jiao, J., Wang, Z.: Fano-chain: the Fano resonances in a nanoparticles chain. J. Mod. Opt. 59(16), 1434-1438 (2012)

29. Wang, M., Cao, M., Chen, X., Gu, N.: Subradiant plasmon modes in multilayer metal-dielectric nanoshells. J. Phys. Chem. C 115(43), 20920-20925 (2011)

30. Liu, S.-D., Yang, Z., Liu, R.-P., Li, X.-Y.: Multiple Fano resonances in plasmonic heptamer clusters composed of split nanorings. ACS Nano 6(7), 6260-6271 (2012)

31. Liu, S.-D., Yang, Y.-B., Chen, Z.-H., Wang, W.-J., Fei, H.-M., Zhang, M.-J., Wang, Y.-C.: Excitation of multiple Fano resonances in plasmonic clusters with $\mathrm{D} 2 \mathrm{~h}$ point group symmetry. J. Phys. Chem. C 117(27), 14218-14228 (2013)
32. Hu, Y., Noelck, S.J., Drezek, R.A.: Symmetry breaking in goldsilica-gold multilayer nanoshells. ACS Nano 4(3), 1521-1528 (2010)

33. Khan, A.D., Miano, G.: Higher order tunable Fano resonances in multilayer nanocones. Plasmonics 8(2), 1023-1034 (2013)

34. Liu, N., Hentschel, M., Weiss, T., Alivisatos, A.P., Giessen, H.: Three-dimensional plasmon rulers. Science 332(6036), 1407-1410 (2011)

35. Zhang, S., Genov, D.A., Wang, Y., Liu, M., Zhang, X.: Plasmoninduced transparency in metamaterials. Phys. Rev. Lett. 101(4), 47401 (2008)

36. Chang, W.-S., Lassiter, J.B., Swanglap, P., Sobhani, H., Khatua, S., Nordlander, P., Halas, N.J., Link, S.: A plasmonic Fano switch. Nano Lett. 12(9), 4977-4982 (2012)

37. Johnson, P.B., Christy, R.-W.: Optical constants of the noble metals. Phys. Rev. B 6(12), 4370 (1972)

38. Wu, D., Jiang, S., Liu, X.: Tunable Fano resonances in threelayered bimetallic $\mathrm{Au}$ and $\mathrm{Ag}$ nanoshell. J. Phys. Chem. C 115(48), 23797-23801 (2011)

39. Prodan, E., Radloff, C., Halas, N., Nordlander, P.: A hybridization model for the plasmon response of complex nanostructures. Science 302(5644), 419-422 (2003)

40. Peña-Rodríguez, O., Pal, U.: Geometrical tunability of linear optical response of silica-gold double concentric nanoshells. J. Phys. Chem. C 114(10), 4414-4417 (2010)

41. Banaee, M.G., Crozier, K.B.: Mixed dimer double-resonance substrates for surface-enhanced Raman spectroscopy. ACS Nano 5(1), 307-314 (2010) 